PROCEEDINGS OF THE AMERICAN MATHEMATICAL SOCIETY

Volume 126, Number 3, March 1998, Pages 801-807

S $0002-9939(98) 04276-2$

\title{
SOME REMARKS ON A BOUNDEDNESS ASSUMPTION FOR MONOTONE DYNAMICAL SYSTEMS
}

\author{
E. N. DANCER
}

(Communicated by Hal L. Smith)

\begin{abstract}
We study the consequences of the assumption that all forward orbits are bounded for monotone dynamical systems. In particular, it turns out that this assumption has more implications than is immediately apparent.
\end{abstract}

In this paper, we consider monotone dynamical systems. In particular, we are interested in the consequences of the assumption that every forward orbit is bounded. We pointed out in [5] (with sketches of proofs) that for monotone dynamical systems this assumption is much stronger than is immediately apparent (and in fact, this assumption frequently implies that the behaviour of our dynamical system largely reduces to its behaviour on an invariant order interval.) Here we improve considerably the result in [5] by showing that on a Banach space the asymptotic behaviour almost completely reduces to the order interval if orbits are order bounded. We use an idea from [7]. We also give a few other simple applications.

Note that the study of this boundedness assumption is of interest because a number of authors have made this assumption without much consideration of its consequences (cf. [10] and [12]). We always state our results for discrete dynamical systems. We discuss very briefly the case of continuous dynamical systems at the end.

\section{THE MAIN RESULT}

Assume that $K$ is a cone in a Banach space $E, T: E \longrightarrow E$ is completely continuous and monotone; that is, $T x-T y \in K$ if $x-y \in K$ and each forward orbit is bounded.

The following is the key result.

Lemma 1. Assume that for each $x \in E, \omega(x)$ is order bounded, $T$ maps order intervals to precompact sets and $z \in E$. Then there exists a fixed point $f$ of $T$ such that $\omega(z) \leq f$.

Proof. Since $\omega(z)$ is order bounded there exists $u \in E$ such that $\omega(z) \leq u$. Since $T(\omega(z))=\omega(z)$, it follows that $\omega(z) \leq T^{i} u$ for all $i$ and hence that $\omega(z) \leq \omega(u)$ since $K$ is closed (in the sense that $a \leq b$ whenever $a \in \omega(z), b \in \omega(u)$ ). Now $\omega(u)$ is order bounded and hence there exists $s \in E$ such that $\omega(u) \leq s$. As before, it follows that $\omega(u) \leq \omega(s)$. Now consider $S=\{x \in E: \omega(z) \leq x \leq \omega(s)\}$. This is the intersection of closed order intervals and hence it is closed and convex. $S$

Received by the editors September 5, 1996.

1991 Mathematics Subject Classification. Primary 47H15.

(C) 1998 American Mathematical Society 
is non-empty since $\omega(z) \leq \omega(u) \leq \omega(s)$. By our assumptions $T(S)$ is precompact. Since $T(\omega(z))=\omega(z), T(\omega(s))=\omega(s)$ and $T$ is monotone, $T(S) \subseteq S$. Thus, by the Schauder fixed point theorem there exists a fixed point $f$ of $T$ in $S$. Hence $f \geq \omega(z)$ as required.

This improves a result in [7]. Note that the order boundedness of $\omega(z)$ from above is clearly necessary.

$K$ is said to be reproducing if $E=K-K$. Note that a cone with non-empty interior is reproducing.

Theorem 1. Assume that $T: E \longrightarrow E$ is completely continuous and monotone, forward orbits are bounded, $\omega(x)$ is order bounded for each $x \in E$ and $T$ maps order intervals to precompact sets.

(i) If $z \in E$, then there exist fixed points $w_{1}, w_{2}$ of $T$ such that $w_{1} \leq \omega(z) \leq w_{2}$.

(ii) Moreover if int $K$ is nonempty, if $\omega(z)$ is not a single point, if $T$ is strongly monotone and if the set of fixed points is bounded, $w_{1}$ and $w_{2}$ can be chosen to be stable and $T$ has at least 3 fixed points.

(iii) If the cone is reproducing and the set of fixed points is bounded, then there exist maximal and minimal fixed points $x_{M}$ and $x_{m}$ of $E$ such that for each $x \in E, T^{n} x \longrightarrow\left[x_{m}, x_{M}\right]$ as $n \longrightarrow \infty$. Moreover, if $x \leq x_{m}, T^{n} x \longrightarrow x_{m}$ as $n \longrightarrow \infty$ while, if $x \geq x_{M}, T^{n} x \longrightarrow x_{M}$ as $n \longrightarrow \infty$.

Proof. (i) This follows immediately from Lemma 1.

(iii) Note that it is convenient to prove (iii) before (ii). Since $F$, the set of fixed points, is bounded, the compactness ensures $F$ is compact. By a simple Zorn's lemma argument, there exist $x_{M} \in F$ weakly maximal; that is, $u>x_{M}$ implies $u \notin F$. We deduce that $x_{M}$ is maximal in the stronger sense that $u \leq x_{M}$ if $u \in F$. Suppose by way of contradiction $g$ is another fixed point of $f$ such that $g \notin\left(-\infty, x_{M}\right]$. Since the cone is reproducing, there exists $u \in E$ such that $u \geq x_{M}$ and $u \geq g$. Now, since $x_{M}$ is a fixed point we see from the monotonicity that $T^{i} u \geq x_{M}$ for all $i$ and hence $\omega(u) \geq x_{M}$. Similarly, $\omega(u) \geq g$. However, by Lemma 1 , there exists a fixed point $f$ such that $\omega(u) \leq f$. Hence $f \geq x_{M}$ and $f \geq g$. This is impossible since $x_{M}$ is weakly maximal and $g \notin\left(-\infty, x_{M}\right]$. Thus $x_{M}$ is maximal in the strong sense. Similarly, we can prove the existence of a minimal fixed point $x_{m}$.

Now suppose $x \in E$. By Lemma 1 and the maximality of $x_{M}, \omega(x) \leq x_{M}$. Similarly, $\omega(x) \geq x_{m}$. Hence $\omega(x) \subseteq\left[x_{m}, x_{M}\right]$. Since $\left\{T^{n} x\right\}_{n=1}^{\infty}$ lies in a compact set, our first claim follows from a simple compactness argument. If $x \leq x_{m}, T^{n} x \leq$ $x_{m}$ for all $n$ by monotonicity and hence $\omega(x) \leq x_{m}$. Thus, by the first part of the statement of (iii), $\omega(x)=\left\{x_{m}\right\}$. Hence $T^{n} x \longrightarrow x_{m}$ as $n \longrightarrow \infty$. The other part of (iii) is proved similarly.

(ii) We first prove the existence of $w_{2}$. The first part of the proof of (iii) showing the existence of the fixed point $x_{M}$ maximal in the stronger sense is still valid. Let $F_{1}$ be the fixed points in $\{u \in F: u \geq \omega(z)\}$ which are strongly order stable from below in the sense of [6], p. 133. We will prove in a moment that $F_{1}$ is non-empty. It is then easy to modify the arguments in Lemmas 3.4-3.7 and Remark 3.2 of [6] to prove that $w_{2}$ can be chosen stable. Note that $F_{2}$ (with the notation of [6]) is non-empty because $x_{M}$ is strongly order stable from above. To see that $x_{M}$ is strongly order stable from above, note that by the maximality of $x_{M}$, it is very easy to see that there are no subsolutions above $x_{M}$ in the order. Thus, by Theorem $1^{\prime}$ and Corollary 1 in [2] (or more precisely the natural analogue of Corollary 1 in the 
strongly increasing case) there are strict supersolutions above $x_{M}$ arbitrarily close to $x_{M}$. Hence $x_{M}$ is strongly order stable from above.

It is easy to see that $F_{1}$ is non-empty if there exist a strict subsolution above $\omega(z)$ in the order. Otherwise, we will prove that a weakly minimal fixed point $\widetilde{z}$ above $\omega(z)$ is in $F_{1}$. This will complete our proof of the existence of the stable fixed point $w_{2}$. It is easy to see from Zorn's Lemma that $\widetilde{z}$ exists. Note that our assumptions ensure $F$ is compact. If $\ell \in \omega(z)$ and $\ell \neq \widetilde{z}, \ell<\widetilde{z}$ and hence by strong monotonicity $T(\ell)<<T(\widetilde{z})=\widetilde{z}$. We prove that there are no supersolutions of $T$ close to $\widetilde{z}$ below $\widetilde{z}$. To see this, note that, if not, there would exist a supersolution $y$ of $T$ with $T(\ell)<<y<\widetilde{z}$. But $T^{n_{i}}(z) \longrightarrow \ell$ as $n_{i} \longrightarrow \infty$ for some sequence $\left\{n_{i}\right\}$. Hence there is an $m$ such that $T^{m}(z) \leq y$. Thus $T^{m+i} z \leq T^{i}(y)$ for $i \geq 0$ which implies that $\omega(z) \leq \widetilde{y}=\lim _{i \longrightarrow \infty} T^{i}(y) \leq y<\widetilde{z}$. Note that the limit exists since $y$ is a supersolution and because of our boundedness assumptions. Since $\widetilde{y}$ is a fixed point, this contradicts the minimality of $\widetilde{z}$. Since there are no supersolutions of $T$ close to $\widetilde{z}$ below $\widetilde{z}$, it follows as before that there are strict subsolutions arbitrarily close to $\widetilde{z}$ below $\widetilde{z}$ (by Theorem $1^{\prime}$ and Corollary 1 in [2]). Hence $\widetilde{z}$ is strongly order stable from below, as required.

The existence of the stable fixed point $w_{1}$ is proved by similar arguments. The existence of another fixed point in $\left[w_{1}, w_{2}\right]$ follows from Theorem 4 in [6] and the stability of $w_{1}$ and $w_{2}$. This completes the proof.

Remarks. 1. The result of (iii) shows that all the interesting dynamics occur in the order interval $\left[x_{m}, x_{M}\right]$ so that the dynamic behaviour really reduces to the behaviour on this interval.

2. We could obtain an analogous theorem when $T$ is only defined on $K$ and $T(K) \subseteq K$. In (iii), we could weaken the reproducing property to $F \subseteq K-K$.

3. The requirement that $\omega(x)$ is order bounded is a restriction. However, one can frequently avoid it in examples by using some form of regularity theory to prove that some iterate $T^{k}$ maps bounded sets to order bounded sets.

4. It would seem unlikely that we can obtain the existence of the stable fixed points without some extra assumptions. We will discuss this result further below.

5. It can be shown that, if $T$ is strongly monotone, a fixed point of $T$ which is isolated from above is strongly order stable from above if and only if it is stable from above. We could use the notion of stable from above rather than strongly order stable from above in the proof of Theorem 1 (ii).

6. If $E$ is a Banach lattice, we can prove Theorem 1 under the assumption that only $\omega(z)$ is order bounded. The proof of this depends on proving that if $C$ is compact, if $T(C)=C$ if $T$ is monotone and completely continuous and if $C$ is order bounded above, then there exists $k$ such that $T k \geq k$ and $k \geq C$. Note that this is non-trivial because a compact order bounded set need not have a least upper bound (cf. [1]). B. de Pagter informed me of this example.

7. We suspect that the order boundedness condition can be weakened. We mention one such result. Assume the conditions of (iii) hold except that we delete the order boundedness condition. We find $x_{M}$ a weakly maximal fixed point as before. We assume that, for each $w \in K$,

$$
y=T(y)+\alpha w
$$

with $0<\alpha \leq 1, y \in\left[x_{M}, \infty\right)$ and $\|y\|$ is large implies that $y \geq w$ (and the analogous condition on $\left(-\infty, x_{m}\right]$ ). Then the conclusion of (iii) holds. (This implies that all 
orbits are order bounded.) If $x \in E$, it suffices to find a strict supersolution $\widetilde{w}$ such that $\widetilde{w} \geq x$ and $\widetilde{w} \geq x_{M}$ (and a strict subsolution $u$ such that $u \leq x$ and $u \leq x_{m}$ ). This suffices because $T^{n} x \in\left[T^{n} u, T^{n} \widetilde{w}\right], T^{n} \widetilde{w} \longrightarrow x_{M}$ as $n \longrightarrow \infty$ and $T^{n} u \longrightarrow x_{m}$ as $n \longrightarrow \infty$. Thus we can use a simple compactness argument to show that $T^{n} x$ converges to $\left[x_{m}, x_{M}\right]$ as $n \longrightarrow \infty$. To construct $\widetilde{w}$, we use a fixed point index argument on $\left[x_{M}, \infty\right)$. Choose $w \in K$ so that $w \geq x-x_{M}$. Now if $R$ is large, $\operatorname{index}_{\left[x_{M}, \infty\right)}\left(T, B_{R}\right)$ is defined since $x_{M}$ is weakly maximal. Now the only fixed point of $T$ in $\left[x_{M}, \infty\right)$ is $x_{M}$ and $x_{M}$ has index 1 since there can be no subsolutions above $x_{M}$ (by Proposition 1 in [2]). Thus $\operatorname{index}_{\left[x_{M}, \infty\right)}\left(T, B_{R}\right)=1$. Fix a large $R$. First assume that $y \neq T(y)+\alpha w$ if $\|y\|=R, y \geq x_{M}$ and $0 \leq \alpha \leq 1$. By homotopy invariance, index $\left.\operatorname{sx}_{M}, \infty\right)\left(T+\alpha w, B_{R}\right)=1$ for $0 \leq \alpha \leq 1$. In particular, there exists $s \in\left[x_{M}, \infty\right)$ such that $s=T s+w$. Since $w \geq 0, s$ is a supersolution and $s \geq T\left(x_{M}\right)+w \geq x_{M}+w \geq x$ (since $T$ is monotone). Hence $s$ is the required supersolution. On the other hand, if $y=T(y)+\alpha w$ with $y \geq x_{M},\|y\|=R$ and $0 \leq \alpha \leq 1$, our assumptions ensure $y \geq w$. It is then easy to see that $y$ is the required supersolution.

Note that the result implies that under our assumptions $x_{M}$ is maximal in the strong sense since, if $b$ is a fixed point of $T, T^{n} b=b$ for all $n$ and $T^{n} b \longrightarrow\left[x_{m}, x_{M}\right]$ as $n \longrightarrow \infty$. Hence $b \leq x_{M}$. Note that the condition (1) can often be established by blow up arguments and that it is nearly always true in the asymptotically linear case. (In particular, it is always true in the asymptotically linear case if int $K$ is non-empty and the asymptotic derivative $A_{\infty}$ maps non-zero elements of $K$ into int $K$.) Here, by asymptotic linearity, we mean $\|x\|^{-1}\left(T(x)-A_{\infty}(x)\right) \longrightarrow 0$ as $\|x\| \longrightarrow \infty$, where $A_{\infty}$ is linear.

Note that if $T$ is asymptotically linear with asymptotic derivative $A_{\infty}$, if $K-K$ is dense in $E$, and if $r\left(A_{\infty}\right)>1$, then it is impossible for all forward orbits to be bounded. It is not difficult to give a direct proof of this by considering iterations starting close to $\alpha \phi_{0}$ where $\alpha$ is large positive, $\phi_{0} \in K$ and $A_{\infty} \phi_{0}=r\left(A_{\infty}\right) \phi_{0}$. Under slightly stronger conditions this can also be deduced by a simple degree argument.

Lastly, we sketch a proof of a version of (ii) for some cones with empty interior. We assume that $K-K$ is dense in $E, T$ is $C^{1}, T^{\prime}(x)$ is a demi-interior operator for every $x \in E$ in the sense of [4, p. 50] and $T$ is quasi strongly increasing in the sense that $T(x)-T(y)$ is strongly quasiinterior to $K$ in the sense of [4] or [10] whenever $x>y$. We assume that the other assumptions of Theorem 1 (ii) hold including that $F$ is bounded. Then the conclusion of Theorem 1 (ii) holds. The proof that there is a stable fixed point of $T$ above $\omega(z)$ is quite similar to the proof of Theorem 1 (ii) except that we replace the argument on p. 134-135 of [6] by an argument very similar to one in Remark 4 on p. 213 of [2]. Note that here it is more convenient to work with the notion of stable from below rather than strongly order stable from below. There are two changes that need to be made (one minor and one rather major). Firstly, since there are strict supersolutions above the weakly maximal fixed point $x_{M}$ and arbitrarily close to $x_{M}, x_{M}$ is stable from above by Theorem 1 in $[2]$.

Secondly, and much less trivially, we need to prove that a minimal fixed point $\widetilde{z}$ above $\omega(z)$ is stable from below. We may assume $\widetilde{z}$ is isolated from below as a fixed point since otherwise the stability from below follows from a simple modification of the proof that (ii) $\Longrightarrow$ (i) in Theorem 1 in [2]. The following more general claim is 
true. If $\omega(z) \leq \widetilde{z}, \widetilde{z}$ is unstable from below and isolated from below and $\omega(z) \neq\{\widetilde{z}\}$, then $\omega(z) \leq z_{1}$ where $z_{1}$ is a maximal fixed point of $T$ below $\widetilde{z}$. Since this clearly contradicts our construction of $\widetilde{z}$, this will complete the proof.

We sketch a proof of our claim. By a change of origin, we can assume $\widetilde{z}=0$. We will assume $r\left(T^{\prime}(0)\right)>1$ and return briefly to the case $r\left(T^{\prime}(0)\right)=1$ at the end of the proof. By applying standard bifurcation theorems in cones to $-K$ at $\lambda=r\left(T^{\prime}(0)\right)^{-1}$ (cf. [3], remarks after Theorem 4, or [4]) we see that there is a curve of solutions $z(\alpha)=\alpha \phi+\widetilde{w}(\alpha), \lambda=\lambda(\alpha)$ of $x=\lambda T(x)$ where $z(\alpha) \in-K$ for small negative $\alpha$ and $\lambda(0)=\left(r\left(T^{\prime}(0)\right)\right)^{-1}<1$. Here $\phi$ is the positive eigenfunction of $T^{\prime}(0)$ corresponding to the eigenvalue $r\left(T^{\prime}(0)\right)$. Since $\lambda(\alpha)<1$, these are supersolutions of $x=T(x)$. Hence $T^{n}(z(\alpha))$ must converge to a fixed point of $T$ less than 0 as $n$ tends to infinity. On the other hand, stable and centre manifold ideas as in Vanderbauwhede [14] imply that if we iterate from $z(\alpha)$ and iterate enough times till $\left\|T^{n}(z(\alpha))\right\|$ is of order 1 (but reasonably small) then $T^{n} z(\alpha)$ will be close to the unstable 1-dimensional invariant manifold $S$ of $T$ tangent to $\phi$ (and becomes closer as $\alpha \longrightarrow 0$ ). Since $T^{n}(z(\alpha))$ are all supersolutions, this implies by a limit argument that the part of $S$ in the direction of $-\phi$ consists entirely of supersolutions and is in $-K$ (and in fact if $S=\{\alpha \phi+\widetilde{w}(\alpha)\}, \alpha \phi+w(\alpha)$ is decreasing in $\alpha$ for small negative $\alpha$.) Assume $m \in \omega(z), m \neq 0$. Since $m=T(v)$ where $v \in \omega(z)$ (and thus $v<0$ ), the demi-interior assumptions imply that $-m$ is demi-interior to $K$ (in the sense of [4]) and hence $\left\{u \in K: 0 \leq u \leq-n m\right.$ for some $\left.n \in Z^{+}\right\}$is dense in $K$. Hence we can find points $s_{n}$ arbitrarily close to $\phi$ in $K$ such that $s_{n} \leq-k_{n} m$ for some $k_{n} \in Z^{+}$. Hence, if $\alpha_{n}$ is sufficiently small and negative, $m \leq \alpha_{n} s_{n}<0$. Hence

$$
T^{k} m \leq T^{k}\left(\alpha_{n} s_{n}\right)
$$

for all $k$. Let us consider more carefully the iterates $T^{k}\left(\alpha_{n} s_{n}\right)$. Since $s_{n}$ is close to $-\phi$, our comments imply that after a large number of iterates $T^{k}\left(\alpha_{n} s_{n}\right)$ is close to a point of $S$ of norm of order 1 . Now points of $S$ must iterate to a fixed point $z_{1}$ of $T$ with $z_{1}<0$ (since points of $S$ are supersolutions). Thus by continuous dependence a large iterate of $\alpha_{n} s_{n}$ must be close to a point of $S$ above $z_{1}$ and close enough to $z_{1}$. (We will specify how close in a moment.) Clearly there are supersolutions arbitrarily close to $z_{1}$ and above $z_{1}$. Thus by Theorem 1 in [2], $z_{1}$ is stable from above and hence $r\left(T^{\prime}\left(z_{1}\right)\right) \leq 1$. We will assume $r\left(T^{\prime}\left(z_{1}\right)\right)=1$. The other case is much easier. Let $\phi_{2}$ be the positive eigenfunction of $T^{\prime}\left(z_{1}\right)$ corresponding to the eigenvalue 1. By a slight variant of the proof that stability on the centre manifold implies stability (cf. [14]) we see that there exist $\epsilon, \delta>0$ such that, if

$$
u \in V=\left\{x \in E:\left\|x-z_{1}\right\| \leq \epsilon \text { and }\left\|\left(\left\|x-z_{1}\right\|\right)^{-1}\left(x-z_{1}\right)-\phi_{2}\right\| \leq \delta\right\},
$$

then $T^{n} u \longrightarrow z_{1}$ as $n \longrightarrow \infty$. Moreover, the iterates move in exponentially towards the part of the centre manifold of $z_{1}$ tangent to $+\phi_{2}$. In particular, the points of $S$ will eventually iterate into $V$. By continuous dependence, we see that the some iterate of $\alpha_{n} s_{n}$ will move into $V$ and hence $T^{k}\left(\alpha_{n} s_{n}\right) \longrightarrow z_{1}$ as $k \longrightarrow \infty$. By (2), any limit point of $\left\{T^{k} m\right\}_{k=1}^{\infty}$ lies in $\left\{x \in E: x \leq z_{1}\right\}$. We now consider iterates of points very close to $m$. Choose $n$ so an iterate of $\alpha_{n} \phi$ is in the interior of the set $V$. By continuous dependence this will be true of any point near $\alpha_{n} \phi$. If $w$ is close to $m, w \leq r$ where $r$ is close to $\alpha_{n} \phi$ and hence $T^{k} w \leq T^{k} r$. But by our results above, $T^{k} r \longrightarrow z_{1}$ as $k \longrightarrow \infty$. Hence any limit point of $T^{k} w$ lies in $\left\{x \in E: x \leq z_{1}\right\}$. But since $m \in \omega(z)$, some iterate of $z$ must lie close to $m$ and hence $\omega(z) \subseteq\left\{x \in E: x \leq z_{1}\right\}$. This contradicts that zero is the minimal fixed 
point above $\omega(z)$. Lastly, if $r\left(T^{\prime}(0)\right)=1$, we need to construct the supersolutions differently. If 0 is unstable from below and isolated from below, then by Theorem 1 in [2] and the remarks after it, index $-K(T, 0)=0$ and the branch of solutions $(\alpha \phi+\widetilde{w}(\alpha), \lambda(\alpha))$ for $\alpha<0$ branching at $(0,1)$ satisfies $\lambda(\alpha)<1$ for small negative $\alpha$ (since index ${ }_{-K}(\lambda T, 0)=1$ if $\lambda<1$ by Theorem 1 in [5] and since the isolatedness ensures $\lambda(\alpha) \neq 1$ for small negative $\alpha)$. This gives the supersolutions. The rest of the proof is unchanged except that we use centre manifolds rather than unstable manifolds.

Note that the information on the centre manifold in the above proof may be useful elsewhere and indeed the above argument is one of the few cases where one has results on dynamic behaviour in cones with empty interior. Note that it is frequently convenient to study partial differential equations in cones with empty interior especially on irregular domains or for mixed boundary conditions. (See the last section in [2], for example.)

Finally, we wish to make some remarks on monotone dynamical systems (that is, the continuous case). Firstly, it is not difficult to prove a natural analogue of Lemma 1 (with fixed point replaced by equilibrium). It is then straightforward to generalize Theorem 1 to this case. Note that for strongly monotone continuous dynamical systems, more is known as in [13] (though Theorem 1 (iii) appears to be new even in this case). Here we obtain some results without strong monotonicity but with some order boundedness conditions. Note also that our results could be used to improve Theorem C in [9] (and prove variants of Theorems 2.3.1 and 2.3.2 in [13] and also prove a discrete time version.) Many more references on the continuous case can be found in [13].

\section{ADDED IN PROOF}

If $K$ has non-empty interior, the assumption in Theorem 1 that $\omega(x)$ is order bounded is a consequence of the other assumptions.

\section{REFERENCES}

1. Y.A. Abramovich and A.W. Wickstead, A compact regular operator without modulus, Proc. Amer. Math. Soc. 116 (1992), 721-726. MR 93a:47038

2. E.N. Dancer, Upper and lower stability and index theory for positive mappings and applications, Nonlinear Analysis TMA 17 (1991), 205-217. MR 92k: 47122

3. E.N. Dancer, Global solution branches for positive mappings, Archives Rational Mech Anal 52 (1973), 181-192. MR 50:5563

4. E.N. Dancer, Multiple fixed points of positive mappings, J. Reine Ang. Math. 371 (1986), 46-66. MR 88b:58020

5. E.N. Dancer, Fixed point calculations and applications, p 303-340 in Topological Nonlinear Analysis (Matzeu and Vignoli, eds.), Birkhauser, Boston, 1995. MR 95i:58006

6. E.N. Dancer and P. Hess, Stability of fixed points for order-preserving discrete-time dynamical systems, J. Reine Ang. Math. 419 (1991), 125-139. MR 92i:47088

7. M. Hirsch, Fixed points of monotone mappings, J. Differential Equations 123 (1995), 171-179. MR 96h:47062

8. M.A. Kransnosel'skii, Positive solutions of operator equations, Noordhoff, Groningen, 1964. MR 31:6107

9. Jiang Ji-Fa, On the global stability of cooperative systems, Bull London Math Soc 26 (1994), 455-458.

10. P. Polacik and I. Terescak, Convergence to cycles as a typical asymptotic behaviour in smooth strongly monotone dynamical systems, Archives Rational Mech Anal 116 (1992), 339-360. MR 93b:58088

11. H.H. Schaefer, Topological vector spaces, Springer, Berlin, 1971. MR 49:7722 
12. H. Smith and H. Thieme, Convergence for strongly order-preserving semiflows, SIAM J. Math Anal 22 (1991), 1081-1101. MR 92m:34145

13. H. Smith, Monotone dynamical systems, Amer. Math. Soc. Providence, 1995. MR 96c:34002

14. A. Vanderbauwhede, Invariant manifolds in infinite dimensions, in Dynamics of Infinite Dimensional Systems, pp 409-420, Springer, Berlin, 1987. MR 89e:47098

School of Mathematics and Statistics, University of Sydney, N.S.W. 2006, Australia

E-mail address: dancer_n@maths.su.oz.au 